\title{
Nanocompuestos de Quitosano Aplicados al Campo de la Medicina Regenerativa. Una Revisión Sistemática
}

\author{
Chitosan Nanocomposites Applied to the Field of Regenerative Medicine. A \\ Systematic Review.
}

\author{
Y. V. Arias-Andrade iD; L. A. Veloza iD; J. C. Sepúlveda-Arias iD \\ DOI: https://doi.org/10.22517/23447214.23411 \\ Artículo de revisión
}

\begin{abstract}
Chitosan is a biopolymer obtained from the chitin found in the exoskeleton of crustaceans, insects, arachnids and the cell wall of fungi. Recent research has confirmed the use of chitosan as a promising biomaterial due to its characteristics such as porous structure, the capacity of chemical modification, high affinity to macromolecules in vivo, among others. The aim of this systematic literature review was to evaluate the applications of chitosan as a biomaterial in regenerative medicine. A literature search was conducted between 2013-2018 in PubMed, Scopus, Web of Science and Embase databases. In this review, the "ToxRTool" (Toxicological data Reliability Assessment Tool) was used to assess the inherent quality or reliability of selected studies at the methodological level. The results showed that the chitosan nanocomposites are being studied mainly using in vitro methodologies $(\mathbf{9 2 . 5 9 \%})$, diverse coupling components, and in multiple applications. However, the lack of methodological information concerning the use of controls for the experiments make reproducibility difficult and constrain the subsequent development of in vivo studies. The ToxRTool was found useful to identify eight studies (29.6\%) as "Reliable without Restrictions" and 19 studies $(70.4 \%)$ as "Reliable with restrictions", indicating that more carefully studies concerning the toxicity of chitosan nanocomposites are required. Chitosan is a versatile biomaterial, which has allowed its incorporation into a wide variety of components, improving its biological, mechanical and physical properties, thus obtaining nanocomposites that are increasingly useful and effective for nanomedicine use.
\end{abstract}

Index Terms - Chitosan; Nanocomposites, Regenerative Medicine.

Resumen-El quitosano es un biopolímero obtenido de la quitina presente en el exoesqueleto de crustáceos, insectos, arácnidos y en la pared celular de los hongos. Investigaciones recientes han confirmado el uso del quitosano como biomaterial prometedor debido a características como estructura porosa, facilidad de modificación química, alta afinidad hacia macromoléculas in vivo,

This manuscript was sent on February 10, 2020 and accepted on December 12, 2020. This is a Systematic review of the literature that was supported by Sistema General de Regalías de Colombia (Proyecto BPIN 2012000100050) and Universidad Tecnológica de Pereira. YVAA was supported by Minciencias Convocatoria 761, Jóvenes Investigadores e Innovadores 2016).

Y. V. Arias-Andrade, Grupo Infección e Inmunidad, Facultad de Ciencias de la Salud, Universidad Tecnológica de Pereira, Carrera 27 \#10-02 Barrio Álamos, Risaralda - Colombia. (yvarias@utp.edu). entre otras. El objetivo de este trabajo fue evaluar las aplicaciones del quitosano como biomaterial en medicina regenerativa. Se realizó una búsqueda de la literatura en el período comprendido entre 2013-2018 en las bases de datos PubMed, Scopus, Web of Science y Embase. En esta revisión, la herramienta "ToxRTool" (Toxicological data Reliability Assessment Tool) se usó para evaluar la calidad y confiabilidad metodológica de los estudios evaluados. Los nanocompuestos de quitosano se están evaluando con metodologías in vitro $(\mathbf{9 2 . 5 9 \% )}$, empleando diversos componentes de acople y en múltiples aplicaciones. Sin embargo, la falta de información metodológica con relación al uso de controles en los estudios dificulta la reproducibilidad de los mismos e introducen limitaciones para el posterior desarrollo de estudios in vivo. La herramienta ToxRTool fue útil para identificar ocho estudios (29.6\%) como "Confiable sin restricciones" y 19 estudios $(\mathbf{7 0 . 4 \% )}$ como "Confiable con restricciones", indicando que se requieren estudios más cuidadosos con relación a la toxicidad de los nanocompuestos de quitosano. El quitosano es un biomaterial versátil lo que ha permitido su incorporación en una amplia variedad de componentes mejorando sus propiedades biológicas, mecánicas y físicas, obteniendo así nanocompuestos cada vez más útiles y eficaces para uso nanomedicina.

Palabras claves - Medicina Regenerativa; Nanocompuestos, Quitosano.

\section{INTRODUCCIÓN}

A medicina regenerativa está orientada al reemplazo de tejidos u órganos dañados por la edad, la enfermedad o el trauma, o alterados por defectos congénitos. Abarca numerosas estrategias que incluyen la generación y el uso de células madre, el uso de biomateriales en ingeniería de tejidos y la producción de órganos artificiales [1]. La medicina regenerativa y la ingeniería de tejidos forman un campo complejo que se extiende desde el estudio a nivel molecular en genética y

L. A. Veloza, Grupo Polifenoles, Facultad de Tecnología, Universidad Tecnológica de Pereira, Carrera 27 \#10-02 Barrio Álamos, Risaralda Colombia. (lveloza@utp.edu).

J. C. Sepúlveda-Arias, Grupo Infección e Inmunidad, Facultad de Ciencias de la Salud, Universidad Tecnológica de Pereira, Carrera 27 \#10-02 Barrio Álamos, Risaralda - Colombia. (jcsepulv@utp.edu). 
biología del desarrollo hasta ingeniería de órganos completos. El objetivo general de reparar o reemplazar tejidos y órganos se ha extendido a una aplicación igualmente importante de diseño de tejidos para pruebas de drogas, modelamiento de enfermedades y medicina de precisión [2].

Los biomateriales son un componente importante en las estrategias actuales de la medicina regenerativa debido a la capacidad que tienen de reproducir las características de la matriz extracelular (MEC) de los tejidos, el comportamiento celular directo, además de favorecer el crecimiento y la diferenciación celular. Las células madre son una alternativa atractiva en la ingeniería de tejidos debido a su capacidad de sintetizar MEC, proliferar, colonizar el andamio y generar el tejido en tres dimensiones. A medida que los vasos sanguíneos crecen en el tejido nuevo, el andamio se degrada y finalmente se regenera el tejido deseado. Para el diseño de un andamio con aplicación biomédica, se debe tener en cuenta la composición del biomaterial, su arquitectura, la mecánica estructural, la topología de la superficie y las propiedades de degradación de este [3].

La nanomedicina puede abordar muchos problemas médicos importantes mediante el uso de materiales estructurados a nanoescala, incluida la interacción de materiales nanoestructurados con sistemas biológicos. Esta rama de la medicina se ha definido como la monitorización, reparación, construcción y control de los sistemas biológicos humanos en los niveles moleculares utilizando nanodispositivos y nanoestructuras. De hecho, los procesos relevantes de los organismos vivos se producen básicamente a escala nanométrica, las unidades biológicas elementales como el ADN, las proteínas o las membranas celulares son de esta dimensión [4]. Los nanocompuestos han atraído mucha atención en la investigación académica y en la industria debido a sus propiedades únicas en comparación con los compuestos convencionales, incluso con un bajo contenido de relleno; estas propiedades provienen del efecto de tamaño nanométrico. La biología celular permite el trabajo con materiales en el rango de 50-200 nm, logrando la absorción de una amplia variedad de células a nanopartículas, donde las modificaciones superficiales de los grupos laterales con carga positiva o negativa de los polímeros, aminoácidos o péptidos/proteínas, aumentan esta absorción [5]. Los biomateriales tienen aplicaciones en medicina, dentro de las que podemos mencionar la ingeniería de tejidos [6-8] y la cicatrización de heridas [7], entre otros.

Actualmente se han preparado una gran variedad de nanocompuestos mediante el uso de diferentes matrices de polímeros y nanorellenos. Los nuevos nanocompuestos biodegradables con volumen modulado y propiedades de superficie han atraído la atención en ingeniería de tejidos y medicina regenerativa [9]. Los nanocompuestos bio-basados y los nanocompuestos biodegradables se han convertido en un tema de interés en los últimos años y se han desarrollado varias matrices adecuadas de polímeros biológicos y biodegradables y sus propiedades han sido mejoradas después de la incorporación de diferentes nanomateriales. Estos sistemas incluyen biopolímeros de recursos naturales, tales como polisacáridos y proteínas, entre los que se destacan ácido poliláctico, polihidroxialcanoatos, fibroína, colágeno, quitosano o biopolímeros de fuentes petroquímicas como el PCL Poly(caprolactona) [10]. Debido a la creciente necesidad de desarrollar materiales poliméricos verdes con estabilidad térmica mejorada, propiedades de barrera a los gases, resistencia y biodegradación, se han generado materiales compuestos basados en polímeros naturales.

Uno de los biomateriales ampliamente utilizado es el quitosano (QS), un biopolímero lineal compuesto de cadenas distribuidas aleatoriamente de $\beta$-(1-4) D-glucosamina y N-acetil- Dglucosamina, obtenido por desacetilación parcial de la quitina, componente estructural de las conchas de crustáceos, exoesqueletos de insectos y pared celular de hongos [11]. El QS exhibe características excepcionales, tales como biocompatibilidad y biodegradabilidad, capacidad de esterilizarse por cualquier método, al igual que propiedades antibacterianas, antifúngicas, mucoadhesivas, analgésicas y hemostáticas. Se ha encontrado que el QS provee una buena adhesión a los tejidos epiteliales y mucosas. Además, está presente en varios sistemas destinados a la administración por vía mucosa de fármacos polares, péptidos, proteínas, vacunas y ADN [12]. Este biopolímero tiene enormes posibilidades estructurales para la modificación química que generan nuevas propiedades importantes para aplicaciones biomédicas [13]. La presente revisión sistemática de la literatura sobre el uso del quitosano como nanocompuesto en medicina regenerativa, tiene como objetivo establecer una relación entre las metodologías empleadas y los avances obtenidos por las ciencias de la salud, a fin de realizar un aporte al avance en la investigación de este biomaterial y mostrar sus usos potenciales.

\section{MATERIALES Y MÉTODOS}

La búsqueda de ésta revisión sistemática se realizó en las bases de datos Pubmed (www.pubmed.com), Scopus (www.scopus.com), Web of Science (www.webofscience.com) y Embase (www.embase.com), empleando la siguiente combinación de términos MeSH "chitosan" AND "biocompatible materials" AND ("tissue engineering" OR "bioengineering" OR "regenerative medicine"), con el fin de determinar los avances en el uso del quitosano como biomaterial para la elaboración de nanocompuestos aplicados en medicina regenerativa.

Se realizó un filtro por año y por idioma tomando solo artículos publicados entre el primero de enero de 2013 y el primero de octubre de 2018, en idioma inglés. Con esta búsqueda se identificaron 231 artículos de los cuales 218 fueron seleccionados por título, resumen y revisión del texto completo. Finalmente, se seleccionaron 27 artículos luego de la aplicación de los criterios de inclusión (estudios experimentales que usen el quitosano, aplicaciones en medicina regenerativa tanto in vitro como in vivo). En la Fig. 1 muestra el diagrama de flujo del proceso de selección de artículos.

Posteriormente, los artículos fueron evaluados mediante la aplicación de la herramienta informática de confiabilidad "ToxRTool" (Toxicological data Reliability Assessment Tool) [14]. Esta herramienta hace uso de una serie de preguntas o 
criterios agrupados, que permiten obtener información de los datos toxicológicos in vitro e in vivo relevante para cada artículo y la califica de forma numérica con el uso de 0 y 1 , donde $0=$ No y $1=$ Sí. La evaluación in vitro consta de 18 criterios agrupados en 5 grupos a saber: identificación de la sustancia de interés, caracterización del sistema aplicado, descripción del diseño del estudio, documentación de resultados del estudio y credibilidad del diseño del estudio y los datos. La evaluación in vivo cuenta con 21 criterios agrupados en los mismos 5 grupos mencionados anteriormente. La ventaja de usar la herramienta "ToxRTool" radica en el hecho de que es posible evaluar en términos de riesgo, la evidencia publicada con relación al uso de los nanocompuestos de quitosano.
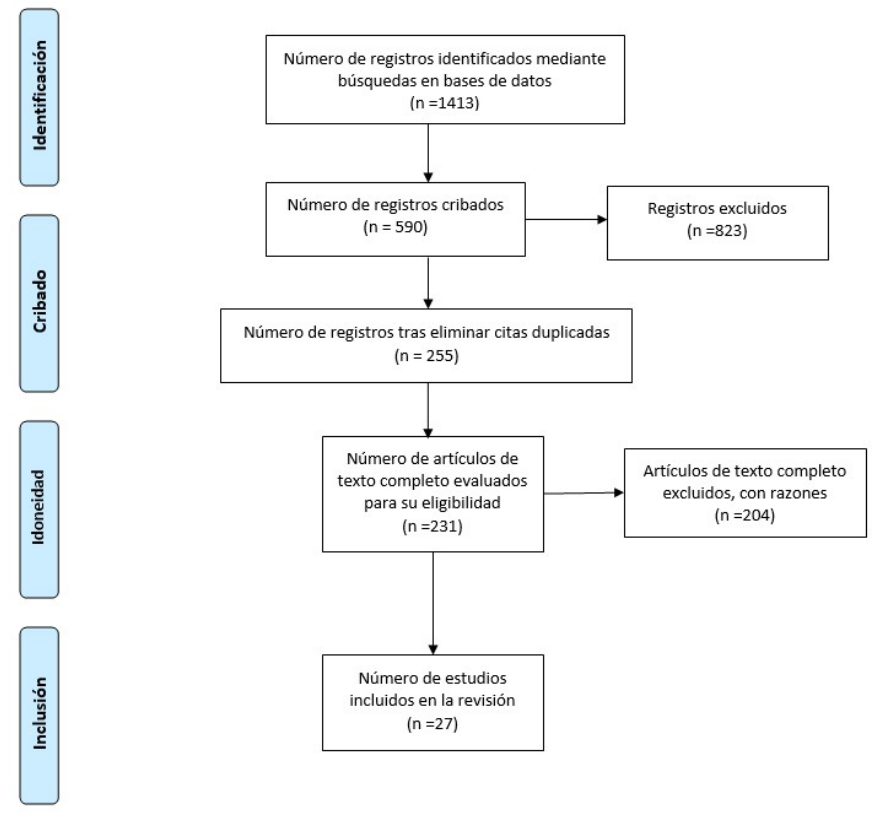

Fig. 1. Flujo de la selección de estudios incluidos en la presente revisión.

\section{RESULTADOS}

En la Tabla I se presentan las características más relevantes de los estudios realizados sobre nanocompuestos de quitosano aplicados en medicina regenerativa, tales como características del andamio, su composición, pruebas biológicas realizadas, usos y las principales conclusiones de los autores.

La mayoría de los nanocompuestos se obtuvieron como nanofibras, seguidos por películas, nanotubos y nanoconjugados multifuncionales, sin embargo, varios estudios no reportan el tipo de nanocompuestos generados. En casi la mitad de los estudios se utilizó el electrospinning como método de obtención de los andamios y se reportan otros métodos como reticulación, mezcla de componentes, precipitación o combinación de dos de éstos métodos.

También se reportan métodos poco comunes como la aerografía, la electrodeposición, la formación de espuma simple, la técnica de capa por capa (LbL) y la fundición de solventes. Se encontraron diferencias en las características fisicoquímicas del quitosano reportadas en los estudios, entre ellos los pesos moleculares que variaron entre 37-1000 Kilodalton (KDa) [15-17], y el grado de desacetilación que varió desde 75 hasta el $96.3 \%$ [18-21]. El 29.6\% de estos estudios emplearon quitosano de peso molecular mediano (150 a $700 \mathrm{KDa})$, el $22.2 \%$ de bajo peso molecular $(<150 \mathrm{KDa})$, el $14.8 \%$ de alto peso molecular $(>700 \mathrm{KDa})$. Algunos artículos emplearon quitosano con otras características o no lo reportaron.

Todos los estudios generaron nanocompuestos de quitosano en combinación con otros biomateriales entre los que se mencionan: hidroxiapatita, alginato, nanotubos de carbono, policaprolactona, gelatina, alcohol polivinílico, óxido de polietileno, ácido poli-láctico, magnetita, mezclas de varios de los mencionados anteriormente o combinaciones con otros biomateriales (péptidos y nanoestructuras semiconductoras, laminina, $\beta$-ciclodextrina, nanopartículas de diamante y plata, o fosfatos cálcicos).

La mayoría de los artículos reportaron ensayos in vitro $(92.6 \%)$ mientras que solo dos (7.4\%) reportan tanto ensayos in vitro como in vivo en ratas. Los ensayos in vitro evaluaron actividad metabólica, adhesión, proliferación, viabilidad celular, actividad antibacteriana, depósito de calcio y las técnicas empleadas fueron amplificación de ADN mediante Reacción en Cadena de la Polimerasa (PCR), análisis de expresión de genes, pruebas inmunohistoquímicas y de inmunofluorescencia. Los ensayos in vivo evaluaron biocompatibilidad mediante inmunohistoquímica. Es importante mencionar que se encontraron discrepancias entre los diferentes artículos en términos de la forma de reportar los controles empleados para los ensayos. Mientras algunos estudios reportan el uso de controles tanto positivos como negativos, cerca de la mitad de los trabajos (48\%) no reportan claramente el tipo de control utilizado o no lo reportan.

Con relación al tipo de uso de los biomateriales basados en quitosano, la mayoría de los artículos reportaron su uso en ingeniería de tejidos (principalmente óseo) y en regeneración de piel, sin embargo, se reportan otros usos como sistemas de administración de medicamentos, regeneración de nervio periférico, regeneración de tráquea o como base para el desarrollo de stent intravasculares.

Solo tres estudios resaltan la importancia del uso exclusivo del quitosano como andamio, mejorando el depósito de fosfato de calcio, las propiedades físicas del andamio y la diferenciación celular [22-24]. La mayoría de los estudios reportan que, en conjunto, todos los componentes que se mezclan para la construcción del andamio refuerzan las características del mismo de forma biomimética y los consideran buenos candidatos como biomateriales.

En las Tablas II y III se muestran las puntuaciones obtenidas en la evaluación de calidad por criterios de los 27 artículos seleccionados utilizando la herramienta "ToxRTool". Para los estudios que realizaron evaluación in vitro, 19 de ellos (70.4\%) mostraron ser confiables, con algunas restricciones mientras que solo 8 (29.6\%) fueron confiables sin restricciones. Para los dos estudios realizados in vivo, solo el trabajo de Nawrotek et al [25] fue confiable sin restricciones mientras que el trabajo de Omidi et [26] no lo fue. 
TABLA II.

RESUltado FinAl De LA Evaluación in vitro

\begin{tabular}{ccc}
\hline \hline Puntuación & $\begin{array}{c}\mathbf{N}^{\circ} \text { de } \\
\text { artículos }\end{array}$ & Conclusión \\
\hline 3 & 0 & $\begin{array}{c}\text { No confiable (puntaje de 12 y } \\
\text { menos) }\end{array}$ \\
2 & 19 & $\begin{array}{c}\text { Confiable con restricciones } \\
\text { (puntaje de 13-17) } \\
\text { Confiable sin restricciones } \\
\text { (puntaje de 18 y superior) }\end{array}$ \\
\hline \hline
\end{tabular}

TABLA III.

Resultado Final De La Evaluación in vivo.

\begin{tabular}{lcl}
\hline \hline \multicolumn{1}{c}{ Autor } & Puntuación & \multicolumn{1}{c}{ Conclusión } \\
\hline (Nawrotek et al., 2016) & 1 & $\begin{array}{l}\text { Confiable sin restricciones } \\
\text { (puntaje de 18 y superior) }\end{array}$ \\
\hline $\begin{array}{l}\text { (Omidi, Yadegari, \& } \\
\text { Tayebi, 2017) }\end{array}$ & 3 & $\begin{array}{l}\text { No confiable (puntaje de 12 y } \\
\text { menos) }\end{array}$ \\
\hline \hline
\end{tabular}

\section{DISCUSIÓN}

Una línea importante de la biomedicina ha sido la medicina regenerativa, que, junto a los aportes ofrecidos por la nanotecnología en la construcción de nuevos biomateriales, han permitido el desarrollo de grandes avances en el sector salud. Es por eso que ha sido un reto de la tecnología construir los nanomateriales cada vez con mayor precisión atómica. Los biomateriales han mostrado gran potencial como soportes para el diseño y reemplazo de tejidos y órganos y es por ello que se ha incrementado el número de estudios en ésta área en los últimos años. Se destaca dentro de estos biomateriales al quitosano, un biomaterial versátil dadas sus características como biocompatibilidad, biodegradabilidad, afinidad química con las moléculas biológicas y su reactividad, las cuales se pueden manipular para convertirlo en un biomaterial atractivo para diversas aplicaciones biomédicas [12].

La presente revisión permitió definir que el principal material generado corresponde a las nanofibras, obtenido generalmente mediante la técnica del electrospinning (electrohilado), el cual es un método atractivo, simple y versátil, capaz de formar nanofibras para la generación de una amplia gama de biopolímeros. Esta permite obtener las fibras con diámetros que van de las sub micras a los nanómetros, rangos en los se pueden encontrar características propias, como la alta relación superficie-área-volumen, flexibilidad en la superficie, alta porosidad, generación de poros interconectados y un rendimiento mecánico superior con otras formas ya conocidas $[27,28]$, lo que hace de las nanofibras un excelente candidato en una variedad de aplicaciones entre ellas ingeniería de tejidos (óseo o traqueal) [22,29], cicatrización de heridas [18], administración de fármacos [30], entre otras aplicaciones.

En cuanto a las características fisicoquímicas del quitosano, ninguno de los estudios analizados hizo relación de éstas con la obtención de los nanocompuestos o sus características. De hecho, cuatro de los artículos no especificaron característica alguna del quitosano empleado [17, 22, 24, 26]. Por otro lado, la mayoría de los estudiosmostraron la utilidad del quitosano de peso molecular medio [20, 26, 29, 23, 31-34], seguida por el de bajo peso molecular [15, 18, 35-38]. Se ha demostrado que las propiedades antioxidantes y antimutagénicas del quitosano están inversamente relacionadas con el peso molecular, para ello tomaron quitosano de alto peso molecular, se degradó enzimáticamente para obtener quitosanos con diferentes pesos moleculares, se evaluaron las actividades de captación de antioxidantes en peróxido de hidrógeno, el radical 2, 2-difenil1-picrilo-hidrazilo y en el ion ferroso quelante que aumentaron significativamente con la disminución del peso molecular del quitosano. Las muestras de quitosano también mostraron fuertes efectos antimutagénicos contra mutágenos directo (4nitroquinolina 1-óxido) e indirectos (benzo $[\alpha]$ pireno) en presencia de la mezcla de enzimas S9 del hígado de rata en Salmonella typhimurium TA98 y TA100 [39] y para observar una alta actividad biológica el grado de desacetilación debe ser mayor a 60\%. Adicionalmente, la degradación está inversamente relacionada con el grado de cristalinidad, la cual se encuentra controlada principalmente por el grado de desacetilación de manera que las formas altamente desacetiladas (DD> 85\%) exhiben una tasa de degradación relativamente baja y pueden durar varios meses en experimentos in vivo, mientras que las formas menos desacetiladas se degradan más rápidamente [40].

El quitosano se considera un buen candidato en la obtención de biomateriales para su aplicación en ingeniería de tejidos y ello es debido a ciertas características tales como: carácter básico por presentar en su estructura grupos amino y grupos hidroxilo que son susceptibles a modificaciones químicas [41], viscosidad, alta solubilidad en diversos medios, capacidad de adhesión a mucosas, comportamiento de polielectrolitos, capacidad para formar películas, capacidad quelante de metales, capacidad de unión a células procariotas y eucariotas, entre otras [42]. Estas características explican porque se evidenció en la literatura la combinación de quitosano con una gran variedad de biomateriales para la obtención de nanocompuestos. Dentro de estos biomateriales podemos mencionar: alginato [37], celulosa [18], colágeno [43], ácido poliláctico [22], gelatina [23], fibroína [34]; polímeros sintéticos como alcohol polivinílico [35], óxido de poliuretano [15], nylon 6 [35], poli(N-isopropilacrilamida) [29]; minerales como la hidroxiapatita [16], magnetita [19], fosfatos cálcicos [34]; macromoléculas como Laminina [33] y $\beta$-ciclodextrina [44], y moléculas bioactivas (péptidos) [36] entre otros. Cabe resaltar que solo dos de los estudios concluyeron que el quitosano mejoró las características físicas del andamio [23, 38], sin embargo, estos estudios no reportan el uso de un control con quitosano puro. Un estudio reporta que el alto contenido de quitosano contribuye a promover la diferenciación celular y la capacidad mielinogénica de las células analizadas [24]. Desafortunadamente, este estudio tampoco reporta el uso del quitosano puro como control. 
Los demás estudios indican que el uso de quitosano mezclado con otros biomateriales mejora las características del andamio, en comparación con el uso del quitosano solo [21, 25, 26]. Desafortunadamente, varios de los estudios reportados no usan un grupo control y, por lo tanto, al no realizarse comparación de resultados entre quitosano solo y combinado, no es posible asegurar que la presencia de quitosano contribuya de manera significativa a la regeneración tisular.

La variedad de aplicaciones del quitosano como biomaterial para la fabricación de nanocompuestos presentada en esta revisión, confirma que ha sido un material ampliamente evaluado para diferentes aplicaciones como la regeneración tisular, la cicatrización de heridas, la administración de fármacos, entre otros $[29,31,35,37,45-50]$ y por lo tanto es un biomaterial con un gran potencial biomédico [12]. Es importante realizar un seguimiento detallado al hecho de que solo dos ensayos se han realizado in vivo (en animales) a pesar del potencial del quitosano demostrado en numerosos ensayos in vitro. Una posible explicación podría ser el hecho de que solo cuatro de los estudios realizados in vitro mostraron el uso de controles tanto positivos como negativos [29, 35, 36, 37], mientras que el resto de los estudios no especificaron el uso de controles. Los controles en los experimentos, tanto in vivo como in vitro son importantes para determinar claramente si un biomaterial tiene o no potencial de aplicación. De hecho, la ausencia de esta información no permite realizar comparaciones y por lo tanto se pone en duda la utilidad del estudio.

Es de tener en cuenta que la liberación de un producto para uso clínico dependerá de la evaluación preclínica rigurosa realizada a través de una serie de pruebas de laboratorio, tanto in vitro como in vivo (en modelos animales como en humanos). La evaluación de la confiabilidad de los estudios que se muestra en las Tablas II y III mostró que más de la mitad de los estudios (70.4\%), resultaron ser confiables pero con restricción y el $29.6 \%$ confiables sin ninguna restricción, indicando que se requiere de un mejor diseño experimental en los trabajos que permitan generar datos reproducibles y confiables que soporten futuros ensayos in vivo con miras a evaluar finalmente en ensayos clínicos, el uso potencial del quitosano en medicina regenerativa [14].

\section{CONCLUSIONES}

Esta revisión permitió mostrar las investigaciones recientes de los biomateriales nanocompuestos aplicados a medicina regenerativa. Sin embargo, se observó que algunas metodologías empleadas, tanto in vitro como in vivo no se describen completamente e incluso no reportan el uso de controles, lo que dificulta la reproducibilidad y la estandarización del método y el avance hacía ensayos preclínicos. El uso de la herramienta "ToxRTool" brinda una aproximación objetiva para evaluar la calidad de los estudios en los que se evalúen moléculas o andamios con potencial uso clínico. El quitosano y sus derivados en combinación con polímeros naturales o sintéticos pueden considerarse como andamios promisorios para uso en medicina regenerativa. El quitosano ha resultado ser un biomaterial versátil que ha permitido su incorporación en una amplia variedad de componentes mejorando propiedades biológicas, mecánicas y físicas, obteniendo así nanocompuestos cada vez más útiles y eficaces para la nanomedicina.

\section{AGRADECIMIENTOS}

Los autores agradecen a Minciencias Convocatoria 761 Jóvenes Investigadores e Innovadores 2016, al Sistema General de Regalías de Colombia (Código BPIN 2012000100050) y a la Universidad Tecnológica de Pereira, por el apoyo financiero.

\section{REFERENCES}

[1] A. Mao et al., "Regenerative medicine: Current therapies and future directions", Proc Natl Acad Sci U S A., vol. 112, no. 47, pp. 1445214459, Nov. 2015, DOI: 10.1073/pnas.1508520112.

[2] H. Wobma et al., "Tissue Engineering and Regenerative Medicine 2015: A Year in Review", Tissue Eng Part B Rev., vol. 22, no. 2, pp. 101-113, Apr. 2016, DOI: 10.1089/ten.teb.2015.0535.

[3] N. Zippel et al., "Biomaterials and Mesenchymal Stem Cells for Regenerative Medicine", Recent Pat Biotechnol., vol. 4, no. 1, pp. 1-22, Sep. 2010, DOI:10.2174/187220810790069497.

[4] J. Gurudutt, "Nanomedicine. A future medicine”, J Today's Bio Sci: Res Rev., vol. 1, no. 1, pp. 179-185, Dec. 2012.

[5] M. Volker et al., "Interaction of Nanoparticles with Cells", Biomacromolecules, vol. 10, no. 9, pp. 2379-2400, Jul. 2009, DOI: 10.1021/bm900266r.

[6] M.S. Enayati et al., "Development of electrospun poly (vinyl alcohol)-based bionanocomposite scaffolds for bone tissue engineering", J Biomed Mater Res A., vol. 106, no. 4, pp. 11111120, Apr. 2018, DOI: 10.1002/jbm.a.36309.

[7] B. Galateanu et al., "In Vitro Studies of Bacterial Cellulose and Magnetic Nanoparticles Smart Nanocomposites for Efficient Chronic Wounds Healing", Stem Cells Int., vol. 2015, pp. 1-10, Apr. 2015, DOI:10.1155/2015/195096.

[8] B. Kaffashi et al., "Poly(E-caprolactone)/triclosan loaded polylactic acid nanoparticles composite: A long-term antibacterial bionanocomposite with sustained release", Int J Pharm., vol. 508, no. 1-2, pp. 10-21, Jul. 2016, DOI: 10.1016/j.ijpharm.2016.05.009.

[9] I. Armentano et al., "Nanocomposites Based on Biodegradable Polymers", Materials (Basel)., vol. 11, no. 5, pp. E795, May. 2018, DOI: $10.3390 / \mathrm{ma} 11050795$.

[10] S.S. Bari et al., "Biodegradable polymer nanocomposites: An overview", Polym Rev., vol. 56, no. 2, pp. 287-328, Sep. 2015. DOI:10.1080/15583724.2015.1118123.

[11] D. Moura et al., "Chitosan nanocomposites based on distinct inorganic fillers for biomedical applications", Sci Technol Adv Mater., vol. 17, no.1, pp. 626-643, Oct. 2016, DOI:10.1080/14686996.2016.1229104.

[12] M. Dash et al., "Chitosan-A versatile semi-synthetic polymer in biomedical applications", Prog Polym Sci., vol. 36, no. 8, pp. 9811014, Aug. 2011, DOI: 10.1016/j.progpolymsci.2011.02.001.

[13] S.K. Shukla et al., "Chitosan-based nanomaterials: A state-of-theart review". Int J Biol Macromol., vol. 59, pp. 46-58, Aug. 2013, DOI: 10.1016/j.ijbiomac.2013.04.043.

[14] K. Schneider et al., "ToxRTool, a new tool to assess the reliability of toxicological data", Toxicol Lett., vol. 189, no. 2, pp. 138-144, Sep. 2009, DOI: 10.1016/j.toxlet.2009.05.013.

[15] A. Aqil et al., "Preparation and characterizations of EGDE crosslinked chitosan electrospun membranes", Clin Hemorheol Microcirc., vol. 60, no. 1, pp. 39-50, Oct. 2014, DOI:10.3233/ch151930.

[16] L. Chen et al., "Synthesis and characterization of chitosanmultiwalled carbon nanotubes/hydroxyapatite nanocomposites for bone tissue engineering", J Mater Sci Mater Med., vol. 24, no. 8, pp. 1843-1851, May. 2013, DOI: 10.1007/s10856-013-4954-x.

[17] B. Derkus et al., "Evaluation of protein immobilization capacity on various carbon nanotube embedded hydrogel biomaterials", Mater 
Sci Eng C Mater Biol Appl., vol. 56, pp. 132-140, Jun. 2015, DOI: 10.1016/j.msec.2015.06.022.

[18] M. Mahdavi et al., "Electrospinning of Nanodiamond-Modified Polysaccharide Nanofibers with Physico-Mechanical Properties Close to Natural Skins", Mar Drugs., vol. 14, no. 7, pp. 128-140, Jul. 2016, DOI: $10.3390 / \mathrm{md} 14070128$.

[19] F. Heidari et al., "Mechanical properties of natural chitosan/hydroxyapatite/magnetite nanocomposites for tissue engineering applications", Mater Sci Eng C Mater Biol Appl., vol. 65, pp. 338-344, Aug. 2016, DOI: 10.1016/j.msec.2016.04.039.

[20] Y. Cheng et al., "Collagen Functionalized Bioactive Nanofiber Matrices for Osteogenic Differentiation of Mesenchymal Stem Cells: Bone Tissue Engineering", J. Biomed Nanotechnol., vol. 10, no. 2, pp. 287-298, Feb. 2014, DOI: 10.1166/jbn.2014.1753.

[21] K. Song et al., "Preparation of the superhydrophobic nano-hybrid membrane containing carbon nanotube based on chitosan and its antibacterial activity", Carbohydr Polym., vol. 130, pp. 381-387, Oct. 2015, DOI: 10.1016/j.carbpol.2015.05.023.

[22] C-C. Lin et al., "Chitosan-coated electrospun PLA fibers for rapid mineralization of calcium phosphate", Int J Biol Macromol., vol. 68, pp. 39-47, Jul. 2014, DOI: 10.1016/j.ijbiomac.2014.04.039.

[23] S. Gomes et al., "Evaluation of nanofibrous scaffolds obtained from blends of chitosan, gelatin and polycaprolactone for skin tissue engineering", Int J Biol Macromol., vol. 102, pp. 1174-1185, May. 2017, DOI: 10.1016/j.ijbiomac.2017.05.004

[24] S. Razavi et al., "Nanobiocomposite of poly(lactide-coglycolide)/chitosan electrospun scaffold can promote proliferation and transdifferentiation of Schwann-like cells from human adiposederived stem cells", J Biomed Mater Res A., vol. 103, no. 8, pp. 2628-2634, Aug. 2015, DOI: 10.1002/jbm.a.35398.

[25] K. Nawrotek et al., "Assessment of degradation and biocompatibility of electrodeposited chitosan and chitosan-carbon nanotube tubular implants", J Biomed Mater Res A., vol. 104, no. 11, pp. 2701-2711, Nov. 2016, DOI:10.1002/jbm.a.35812.

[26] M. Omidi et al., "Wound dressing application of $\mathrm{pH}$-sensitive carbon dots/chitosan hydrogel", RSC Adv., vol. 7, pp. 10638-10649, Jan. 2017, DOI:10.1039/c6ra25340g.

[27] L.M.D. Sánchez et al., "Electrospinning: La era de las nanofibras", Rev Iberoam Polim., vol. 14, no. 1, pp. 10-27, Jan. 2013.

[28] H-W. Tong et al., "A new nanofiber fabrication technique based on coaxial electrospinning", Mater Lett., vol. 66, no. 1, pp. 257-260, Sep. 2012, DOI: 10.1016/j.matlet.2011.08.095.

[29] C. Mahoney et al., "Electrospun nanofibers of poly( $\varepsilon$ caprolactone)/depolymerized chitosan for respiratory tissue engineering applications", J Biomater Sci Polym Ed., vol. 27, no. 7, pp. 611-625, Mar. 2016, DOI:10.1080/09205063.2016.1144454.

[30] H. Yuan et al., "Regulating drug release from $\mathrm{pH}$ - and temperature-responsive electrospun CTS-g-PNIPAAm/poly (ethylene oxide) hydrogel nanofibers", Biomed Mater., vol. 9, no. 5, pp. 1-10, Aug. 2014, DOI:10.1088/1748-6041/9/5/055001.

[31] C. Sharma et al., "Fabrication and characterization of novel nanobiocomposite scaffold of chitosan-gelatin-alginate-hydroxyapatite for bone tissue engineering", Mater Sci Eng C Mater Biol Appl., vol. 64, no. 1, pp. 416-427, Mar. 2016, DOI: 10.1016/j.msec.2016.03.060

[32] J.M. Silva et al., "Nanostructured Hollow Tubes Based on Chitosan and Alginate Multilayers", Adv Healthcare Mater., vol. 3, no. 3, pp. 433-440, Aug. 2013, DOI:10.1002/adhm.201300265.

[33] L. He et al., "Surface Modification of PLLA Nano-scaffolds with Laminin Multilayer by LbL Assembly for Enhancing Neurite Outgrowth", Macromol Biosci, vol. 13, no. 11, pp. 1601-1609, Nov. 2013, DOI:10.1002/mabi.201300177.

[34] W.I. Abdel-Fattah et al., "Tailoring the properties and functions of phosphate/silk/Ag/chitosan scaffolds", Mater Sci Eng C Mater Biol Appl., vol. 54, no. 1, pp. 158-168, May. 2015, DOI: 10.1016/j.msec.2015.05.015.

[35] S.K. Bhullar et al., "Antibacterial activity of combination of synthetic and biopolymer non-woven structures", J Complement Integr Med., vol. 12, no. 4, pp. 289-294, Jun. 2015, DOI: 10.1515/jcim-2015-0027.

[36] A.A. Mansur et. al., "Bioengineered quantum dot/chitosantripeptide nanoconjugates for targeting the receptors of cancer cells", Int J Biol Macromol., vol. 82, no. 1, pp. 780-789, Oct. 2016, DOI: 10.1016/j.ijbiomac.2015.10.047.
[37] [37] W-W. Hu et al., "Coelectrospinning of chitosan/alginate fibers by dual-jet system for modulating material surfaces", Carbohydr Polym., vol. 95, no. 2, pp. 716-727, Feb. 2013, DOI: 10.1016/j.carbpol.2013.02.083.

[38] E. Bolaina et al., "Electrospun polycaprolactone/chitosan scaffolds for nerve tissue engineering: physicochemical characterization and Schwann cell biocompatibility", Biomed Mater., vol. 12, no. 1, pp. 015008, Dec. 2016, DOI: 10.1088/1748-605x/12/1/015008.

[39] S-H. Chang et al., "Effects of chitosan molecular weight on its antioxidant and antimutagenic properties", Carbohydr Polym., vol 181, no. 1, pp. 1026-1032, Nov. 2017, DOI: 10.1016/j.carbpol.2017.11.047.

[40] I-Y. Kim et al., "Chitosan and its derivatives for tissue engineering applications", Biotechnol. Adv., vol. 26, no. 1, pp. 1-21, Aug. 2007, DOI: 10.1016/j.biotechadv.2007.07.009.

[41] I.R. Zwirtes De Oliveira et al., "Development of a biosensor based on gilo peroxidase immobilized on chitosan chemically crosslinked with epichlorohydrin for determination of rutin", J Pharm Biomed Anal., vol. 41, no. 2, pp. 366-372, Feb. 2006, DOI: 10.1016/j.jpba.2005.12.019

[42] S.K. Shukla et al., "Chitosan-based nanomaterials: A state-of-theart review", Int. J. Biol. Macromol., vol. 59, no. 1, pp. 46-58, Apr. 2013, DOI: 10.1016/j.ijbiomac.2013.04.043.

[43] F. Zou et al., "Mechanical enhancement and in vitro biocompatibility of nanofibrous collagen-chitosan scaffolds for tissue engineering”, J Biomater Sci Polym Ed., vol. 28, no. 18, pp. 2255-2270, Oct. 2017, DOI: 10.1080/09205063.2017.1392672.

[44] M. Shakir et al., "Nano-hydroxyapatite/ $/$-CD/chitosan nanocomposite for potential applications in bone tissue engineering", Int. J. Biol. Macromol., vol. 93, no. Pt A, pp. 276289, Aug. 2016, DOI: 10.1016/j.ijbiomac.2016.08.046.

[45] P. Stafiej et al., "Adhesion and metabolic activity of human corneal cells on PCL based nanofiber matrices", Mater. Sci. Eng. C., vol. 71 , no. $1, \quad$ pp. 764-770, Oct. 2016, DOI: 10.1016/j.msec.2016.10.058.

[46] M. Koosha et al., "Electrospinning, mechanical properties, and cell behavior study of chitosan/PVA nanofibers", J Biomed Mater Res A., vol. 103, no. 9, pp. 3081-3093, Feb. 2015, DOI: 10.1002/jbm.a.35443.

[47] S. Deepthi et al., "An overview of chitin or chitosan/nano ceramic composite scaffolds for bone tissue engineering", Int $J$ Biol Macromol., vol. 93 (Pt B), pp. 1338-53, Mar 2016, DOI: doi: 10.1016/j.ijbiomac.2016.03.041.

[48] J. Lewandowska-Łańcucka et al., "Bioactive hydrogel-nanosilica hybrid materials: a potential injectable scaffold for bone tissue engineering", Biomed Mater., vol. 10, no. 1, pp. 1-17, Feb. 2015, DOI: 0.1088/1748-6041/10/1/015020.

[49] A. Oryan et al., "Effectiveness of chitosan scaffold in skin, bone and cartilage healing", Int J Biol Macromol., vol. 104 (Pt A), pp. 10031011, Nov. 2017, DOI: 10.1016/j.ijbiomac.2017.06.124.

[50] H. C. Navia et al., "Biomateriales y su aplicación en el campo de la salud" en Biotecnología y sus aplicaciones en el sector salud, $1^{\mathrm{a}} \mathrm{Ed}$. Pereira, Colombia. Editorial Universidad Tecnológica de Pereira, 2020, pp. 285-318. 
TABLA I.

Estudios Sobre NANOCOMPUESTOS BASADOS EN Qs APLICADOS A LA BIOMEDICINA.

\begin{tabular}{|c|c|c|c|c|}
\hline Autores & Tipo de nanocompuesto & Usos del soporte & Ensayos realizados & Conclusiones \\
\hline (Stafiej et al., 2017) & $\begin{array}{l}\text { Nanofibras de } 144 \mathrm{~nm} \text { obtenidas por } \\
\text { electrospinning: QS (bajo PM) +PGS. }\end{array}$ & Adherencia celular in vitro. & $\begin{array}{l}\text { In vitro: Actividad metabólica, cultivo celular, } \\
\text { evaluación de crecimiento y adherencia celular. } \\
\text { Células epiteliales corneales humanas y } \\
\text { queratinocitos corneales humanos. }\end{array}$ & $\begin{array}{l}\text { Todas las matrices producidas proporcionaron buena } \\
\text { biocompatibilidad y todas las mezclas de polímeros y } \\
\text { estructuras mostraron un buen rendimiento como } \\
\text { sustrato para el crecimiento celular. }\end{array}$ \\
\hline (Bhullar et al., 2015) & $\begin{array}{l}\text { Películas y estructuras nanofibrosas } \\
\text { obtenida aerógrafía: (PVA) + QS (PM=100 } \\
\text { KDa) + nylon-6. }\end{array}$ & $\begin{array}{l}\text { Sistemas de administración de medicamentos y } \\
\text { parches transdérmicos. }\end{array}$ & In vitro: Actividad antibacteriana. & $\begin{array}{l}\text { La combinación de PVA con QS fue más bactericida } \\
\text { o bacteriostática que la del nylon-6 combinado con } \\
\text { QS y ácido fórmico. La combinación de PVA con QS } \\
\text { parece ser un agente antimicrobiano de amplio } \\
\text { espectro. }\end{array}$ \\
\hline $\begin{array}{l}\text { (Nawrotek et al., } \\
\text { 2016) }\end{array}$ & $\begin{array}{l}\text { Implantes nanotubulares de hidrogeles } \\
\text { obtenidos por electrodeposición: QS } \\
(\mathrm{V}=400 \mathrm{mPa} * \mathrm{~s}) / \mathrm{QS}\left(\mathrm{V}=400 \mathrm{mPa}^{*} \mathrm{~s}\right)+ \\
\text { carbono. }\end{array}$ & Ingeniería de tejidos. & $\begin{array}{l}\text { In vitro: Ensayo citotóxico, imágenes celulares, } \\
\text { estudios de degradación. Células del } \\
\text { hipocampo murino. } \\
\text { In vivo: Prueba de biocompatibilidad. } \\
\text { (15 ratas albinas adultas Sprague Dawley, } \\
\mathrm{n}=5 \text { ). }\end{array}$ & $\begin{array}{l}\text { La electrodeposición presentó ventajas mientas que } \\
\text { los nanocompuestos tuvieron buena adhesión, } \\
\text { proliferación y viabilidad, además mantienen su } \\
\text { estabilidad estructural y no mostraron síntomas de } \\
\text { inflamación aguda. }\end{array}$ \\
\hline $\begin{array}{l}\text { (Mansur, de Carvalho, } \\
\quad \& \text { Mansur, 2016) }\end{array}$ & $\begin{array}{l}\text { Nanoconjugados multifuncionales de } 18-51 \\
\text { nm obtenidos por reticulación y por una } \\
\text { ruta acuosa en un matraz de reacción: QS } \\
\text { (PM= (60-70 KDa, DD=96.1\%) + péptido } \\
\text { RGD + núcleos de puntos cuánticos de } \\
\text { cadmio sulfuro (CdS). }\end{array}$ & $\begin{array}{l}\text { Identificar los receptores de integrina } \alpha v \beta 3 \text { de las } \\
\text { células cancerosas. }\end{array}$ & $\begin{array}{l}\text { In vitro: Ensayo de citotoxicidad (MTT) e } \\
\text { imágenes de inmunofluorescencia. Línea } \\
\text { celular de sarcoma humano y riñón } \\
\text { embrionario humano. }\end{array}$ & $\begin{array}{l}\text { Los nanobiomateriales QS-RGD reconocen } \\
\text { eficazmente los receptores de integrina específicos del } \\
\text { cáncer en biointerfaces celulares. Los conjugados } \\
\text { CdS-QS-RGD resultaron ser citocompatibles y tienen } \\
\text { actividad fluorescente en el rango visible. }\end{array}$ \\
\hline $\begin{array}{l}\text { (Lin, Fu, Lin, Yang, \& } \\
\text { Gu, 2014) }\end{array}$ & $\begin{array}{l}\text { Nanofibras de } 365.35-386.76 \mathrm{~nm} \text { obtenidas } \\
\text { por la técnica capa por capa (LBL): HA + } \\
\text { PLA + QS.) }\end{array}$ & Ingeniería de tejido de hueso. & $\begin{array}{l}\text { In vitro: Proliferación celular, ensayo de } \\
\text { actividad ALP. Células de osteosarcoma } \\
\text { humano. }\end{array}$ & $\begin{array}{l}\text { La nanofibra de PLA recubierta con QS } 4 \% \text { mostró } \\
\text { más cristales de fosfato de calcio, además la } \\
\text { mineralización de HA en QS recubierta con fibra de } \\
\text { PLA puede prepararse de manera simple. }\end{array}$ \\
\hline
\end{tabular}


TABLA I.

Continuación

Continuación

$$
\text { Tipo de nanocompuesto }
$$

Usos del soporte

Ensayos realizados

Conclusiones

Nanofibras de $200 \mathrm{~nm}$ obtenidas por electrospinning coaxial: PLLA + QS $(\mathrm{PM}=200 \mathrm{KDa}, \mathrm{DD}=85 \%)$, reticulada con genipina y modificada por heparina.

Biomaterial en un stent intravascular.

In vitro: Anticoagulación sanguínea (conejos), experimentos de flujo de sangre, proliferación celular (Ensayo MTT). Células de médula ósea humana.

Nanofibras de 293-322 nm obtenidas por electros $\mathrm{KDa})+$ ALG. $/ \mathrm{QS}(\mathrm{PM}=50-190$ $\mathrm{KDa})+\mathrm{ALG}+\mathrm{PEO}$ )

(Cheng et al., Nanofibras de 700-850 nm obtenidas 2014) por electrospinning: $\mathrm{PCL}+\mathrm{QS}(\mathrm{PM}=$ por electrospinning: PCL + QS
190-310 KDa, DD= 75-85 \%).

Ingeniería de tejidos.

Ingeniería de tejidos sin especificación.

In vitro: Ensayo de biocompatibilidad (Ensayo Lactato deshidrogenasa (LDH) y MTT. Células de riñón embrionario humano.

In vitro: Adhesión y propagación, proliferación, ensayo de fosfatasa alcalina (ALP), ensayo de deposición de calcio y

análisis por PCR del marcador osteogénico. Células estromales de médula ósea de rata.

In vitro: Adherencia y proliferación

$\begin{array}{cl}\text { (Koosha \& } & \begin{array}{l}\text { Esferas nanofibrosas de 172-257 nm } \\ \text { obtenidas por electrospinning: PVA + }\end{array}\end{array}$ $\mathrm{QS}\left(\mathrm{V}=50 \mathrm{~Pa}^{*} \mathrm{~s}\right.$. $\left.\mathrm{DD}=88 \%\right)$

No especifica

celular Células de fibroblasto de ratón.

In vitro: Estudio de toxicidad celular y

Conklin,

Nanofibras de 80-300 $\mathrm{nm}$ obtenidas Waterman, Sankar, \& Bhattarai, 2016) por electrospinning: PCL + QS (190

$\mathrm{KDa})$.
Regeneración del tejido traqueal. ensayo de Lactato Deshidrogenasa traqueobronquial porcino.
(LDH). Células del epitelio
Las propiedades mecánicas de las nanofibras mejoraron por el PLA y la reticulación superficial por genipina. Se mejoró la biocompatibilidad de la fibra de QS con

heparina, además de exhibir una gran actividad anticoagulante.

Las fibras compuestas demuestran una mejor biocompatibilidad que las fibras puras. El sistema de coelectrospinning puede controlar la relación de fibra, lo que debería proporcionar un enfoque para modular dinámicamente las propiedades de superficies.

La inmovilización del colágeno en las nanofibras podría aplicarse a otras proteínas y factores de crecimiento. Se observó mayor adhesión y proliferación en la nanofibra con colágeno, también resultaron ser favorables para la expresión del biomarcador específico de osteoblastos y mineralización de la matriz.

Los resultados del estudio de comportamiento celular indicaron que la estera nanofibrosa estabilizada con calor QS / PVA 30/70 fue capaz de soportar la unión y proliferación de firboblastos..

Las nanofibras de PCL /QS exhibieron uniformidad competente, integridad estructural, propiedades mecánicas adecuadas y compatibilidad celular, además con células PTBE mostraron una respuesta topográfica positiva. 
TABLA I.

Continuación

\begin{tabular}{|c|c|c|c|c|}
\hline Autores & Tipo de nanocompuesto & Usos del soporte & Ensayos realizados & Conclusiones \\
\hline $\begin{array}{l}\text { (Bolaina-Lorenzo } \\
\text { et al., 2017) }\end{array}$ & $\begin{array}{l}\text { Nanofibras de } 110-240 \mathrm{~nm} \text { obtenidas } \\
\text { por electrospinning: } \mathrm{PCL}+\mathrm{QS} \\
(\mathrm{PM}=54.9 \mathrm{KDa}, \mathrm{DD}=78 \%) \text { reticulado } \\
\text { con glutaraldehído. }\end{array}$ & $\begin{array}{l}\text { Ingeniería de los tejidos nerviosos } \\
\text { periféricos. }\end{array}$ & $\begin{array}{l}\text { In vitro: Prueba de citotoxicidad, } \\
\text { proliferación, viabilidad celular (MTT), } \\
\text { análisis inmunoquímicoFibroblastos de } \\
\text { ratón y células de Schwann. }\end{array}$ & $\begin{array}{l}\text { El diámetro de la nanofibra disminuyó con un } \\
\text { contenido creciente de QS. Las células } \\
\text { Schwann se adhirieron al soporte que contenía } \\
\text { QS al 5\% en peso, exhibiendo la mayor } \\
\text { proliferación. }\end{array}$ \\
\hline $\begin{array}{l}\text { (Gomes, } \\
\text { Rodrigues, Martins, } \\
\text { Henriques, \& Silva, } \\
\text { 2017) }\end{array}$ & $\begin{array}{l}\text { Nanofibras de } 424-1236 \mathrm{~nm} \text { obtenidas } \\
\text { por electrospinning: PCL + QS ( } 500 \\
\text { KDa) + GEL. }\end{array}$ & Ingeniería de tejidos de piel. & $\begin{array}{l}\text { In vitro: Adhesión, proliferación celular, } \\
\text { viabilidad celular, estudios de } \\
\text { inmunocitoquímica y morfología. } \\
\text { Fibroblastos fetales humanos. }\end{array}$ & $\begin{array}{l}\text { Los andamios QS / PCL / GEL tienen mejores } \\
\text { propiedades físicas, mientras que los andamios } \\
\text { PCL / GEL soportan una mayor adhesión } \\
\text { celular. }\end{array}$ \\
\hline $\begin{array}{l}\text { (Derkus, Emregul, } \\
\text { \& Emregul, 2015) }\end{array}$ & $\begin{array}{l}\text { Electrodos nanocompuestos obtenidos } \\
\text { por mezcla de polímeros y } \\
\text { reticulación: GEL + ALG + QS + } \\
\text { CNT. }\end{array}$ & Sistemas de detección. & No se realizaron ensayos biológicos & $\begin{array}{l}\text { Se obtuvieron eficiencias de inmovilización } \\
\text { superiores con la adición de nanotubos de } \\
\text { carbono, especialmente en las estructuras de } \\
\text { GEL-QS debido a la presencia de grupos } \\
\text { estructurales activos. }\end{array}$ \\
\hline $\begin{array}{l}\text { (Sharma, Dinda, } \\
\text { Potdar, Chou, \& } \\
\text { Mishra, 2016) }\end{array}$ & $\begin{array}{l}\text { Andamio nanobiocompuesto obtenido } \\
\text { por formación de espuma simple: QS } \\
\text { (PM=100-300 KDa) + GEL, ALG } \\
\text { +nHA }\end{array}$ & Ingeniería de tejidos óseos. & $\begin{array}{l}\text { In vitro: Viabilidad celular, proliferación, } \\
\text { adhesión, tinción histológica y estudios } \\
\text { de expresión génica. Línea celular de } \\
\text { osteoblastos. }\end{array}$ & $\begin{array}{l}\text { El andamio posee excelentes propiedades de } \\
\text { hidrofobicidad y biodegradabilidad, posee } \\
\text { buena estabilidad mecánica. Adicionalmente, } \\
\text { resultó ser un buen andamio para la adhesión y } \\
\text { proliferación de osteoblastos. }\end{array}$ \\
\hline (Zou et al., 2017) & $\begin{array}{l}\text { Nanofibras obtenidas por } \\
\text { electrospinning: CG+ QS (1000 KDa, } \\
\text { D=85\%) + PVA + TPU. }\end{array}$ & $\begin{array}{l}\text { Reparación de tejidos y aplicaciones } \\
\text { biomédicas. }\end{array}$ & $\begin{array}{l}\text { In vitro: ensayos de biocompatibilidad } \\
\text { (MTT). Células endoteliales porcinas. }\end{array}$ & $\begin{array}{l}\text { La tracción mejoró con el contenido de CG en } \\
\text { las fibras. El módulo de Young, la resistencia a } \\
\text { la rotura y la elongación de rotura de los } \\
\text { materiales se intensificaron con PVA. Los } \\
\text { materiales podrían proporcionar un entorno } \\
\text { adecuado para la adhesión celular y la } \\
\text { proliferación. }\end{array}$ \\
\hline $\begin{array}{l}\text { (Omidi, Yadegari, } \\
\text { \& Tayebi, 2017) }\end{array}$ & $\begin{array}{l}\text { Película nanocompuesta obtenidas por } \\
\text { el método de fundición con solvente: } \\
\text { QS (NA) + carbono (CD). }\end{array}$ & $\begin{array}{l}\text { Monitoreo del pH durante el proceso de } \\
\text { curación de la herida. }\end{array}$ & $\begin{array}{l}\text { In vitro: Viabilidad celular, sensibilidad } \\
\text { al } \mathrm{pH} \text {. Fibroblastos murinos. } \\
\text { In vivo: Estudio de cicatrización de } \\
\text { heridas (modelo de rata). }\end{array}$ & $\begin{array}{l}\text { Los nanocompuestos de QS / CD eran } \\
\text { biocompatibles y no tóxicos con propiedades } \\
\text { antibacterianas efectivas, las propiedades } \\
\text { mecánicas del nanocompuesto de QS / CD se } \\
\text { mejoraron mediante la adición de los CD. }\end{array}$ \\
\hline
\end{tabular}


TABLA I.

Continuación

\begin{tabular}{|c|c|c|c|c|}
\hline Autores & Tipo de nanocompuesto & Usos del soporte & Ensayos realizados & Conclusiones \\
\hline $\begin{array}{l}\text { (Heidari et al., } \\
\text { 2016) }\end{array}$ & $\begin{array}{l}\text { Nanocompuestos: QS }(\mathrm{DD}=75 \%)+ \\
\mathrm{HA}+\text { magnetita. }\end{array}$ & No especifica & $\begin{array}{l}\text { In vitro: Ensayos de proliferación celular } \\
\text { y viabilidad celular. Células madre } \\
\text { mesenquimales humanas. }\end{array}$ & $\begin{array}{l}\text { Todos estos compuestos poseen } \\
\text { biocompatibilidad in vitro, la adición de HA y } \\
\text { magnetita a la matriz de quitosano puede } \\
\text { mejorar notablemente las propiedades } \\
\text { mecánicas del QS. }\end{array}$ \\
\hline $\begin{array}{l}\text { (Razavi et al., } \\
\text { 2015) }\end{array}$ & $\begin{array}{l}\text { Nanofibras obtenidas por } \\
\text { electrospinning: QS + PLGA. }\end{array}$ & $\begin{array}{l}\text { Regeneración de tejidos nerviosos } \\
\text { periféricos dañados }\end{array}$ & $\begin{array}{l}\text { In vitro: Aislamiento y cultivo de células } \\
\text { madre, inducción de ADSC en células S- } \\
\text { like, estudio morfológico de células, } \\
\text { viabilidad de las células S-like, técnica } \\
\text { de inmunocitoquímica. . }\end{array}$ & $\begin{array}{l}\text { El alto contenido de QS y la alineación de } \\
\text { nanofibras en el andamio biocompuesto ( } 70 \text { / } \\
\text { 30A) pueden promover la diferenciación y la } \\
\text { capacidad mielinogénica de las células de S- } \\
\text { like inducidas por las ADSC humanas. }\end{array}$ \\
\hline $\begin{array}{l}\text { (Shakir, Jolly, } \\
\text { Khan, Rauf, \& } \\
\text { Kazmi, 2016) }\end{array}$ & $\begin{array}{l}\text { Nanocompuestos obtenidos por co- } \\
\text { precipitación: } \beta \text {-ciclodextrina }(-\mathrm{CD})+ \\
\text { nHA + QS (DD=85\%) }\end{array}$ & Ingeniería de tejidos óseos. & $\begin{array}{l}\text { In vitro: Inmersión en fluidos corporales } \\
\text { simulados (SBF): prueba de } \\
\text { mineralización, estudio de degradación, } \\
\text { ensayo (MTT), evaluaciones } \\
\text { antibacterianas, evaluación de } \\
\text { hemocompatibilidad, prueba de } \\
\text { hemólisis, adherencia de plaquetas, y } \\
\text { ensayo de actividad de la fosfatasa } \\
\text { alcalina (ALP). Células de osteosarcoma } \\
\text { humano. }\end{array}$ & $\begin{array}{l}\text { Los nanocomposites n-HA / -CD / QS- (RT, } \\
\text { HT, LT) tuvieron mejor hemocompatibilidad } \\
\text { con propiedad bacteriana en comparación con } \\
\text { QS/ n-HA. Los parámetros mecánicos } \\
\text { mejorados de n-HA / -CD / CS-LT en } \\
\text { comparación con n-HA / -CD / QS- (RT, HT) } \\
\text { y QS / n-HA. }\end{array}$ \\
\hline (Silva et al., 2014) & $\begin{array}{l}\text { Tubos multicapa huecos } \\
\text { nanoestructurados obtenidos por } \mathrm{LbL} \\
\text { (capa por capa) y lixiviación de } \\
\text { plantillas: QS (PM=190-310 KDa, } \\
\text { DD=88\%) +ALG. }\end{array}$ & Ingeniería de tejidos. & $\begin{array}{l}\text { In vitro: Adhesión, morfología, ensayo } \\
\text { de viabilidad celular y cuantificación de } \\
\text { ADN. Fibroblastos murinos. }\end{array}$ & $\begin{array}{l}\text { Generación de nuevas perspectivas en el } \\
\text { desarrollo de dispositivos híbridos para } \\
\text { enfoques vasculares. La característica más } \\
\text { prometedora de los tubos multicapa es la } \\
\text { capacidad de ajustar diferentes propiedades en } \\
\text { una estructura, creando nuevos materiales } \\
\text { multifuncionales. }\end{array}$ \\
\hline (Aqil et al., 2015) & $\begin{array}{l}\text { Membranas nanofibrosas de }(200- \\
800) \text { nm obtenidas por } \\
\text { electrospinning seguida de } \\
\text { reticulación química mediada por } \\
\text { calor: QS (PM= } 37 \mathrm{KDa}, \mathrm{DD}=82 \%) \\
\text { +EGDE + PEO }\end{array}$ & Ingeniería de tejidos. & $\begin{array}{l}\text { In vitro: Apego y morfología de las } \\
\text { células. Fibroblastos de piel. Células } \\
\text { endoteliales. }\end{array}$ & $\begin{array}{l}\text { Los fibroblastos de piel y las células } \\
\text { endoteliales mostraron una buena unión, } \\
\text { proliferación y viabilidad en membranas } \\
\text { electrohiladas reticuladas. Los resultados } \\
\text { indican una buena biocompatibilidad y } \\
\text { naturaleza no tóxica de la membrana } \\
\text { resultante. }\end{array}$ \\
\hline
\end{tabular}


TABLA I

Continuación

(Song, Gao,

Cheng, \& Xie,

Yuan, Li, Liang,

Lou, \& Zhang,

(He et al., 2013)

por electrospinning y método capa

por capa (LBL): PLLA + QS

$(\mathrm{DD}=85 \%)+\mathrm{LN}+$ Poli

(etilenoimina).

(Chen, Hu, Shen,

\& Tong, 2013)

Nanocompuestos obtenidos por

precipitación in situ: CNT + nHA QS

$(\mathrm{PM}=1000 \mathrm{KDa})$

Nanopartículas obtenidas por mezcla

(Abdel-Fattah, de componentes: HA + octa fosfatos

Sallam, Diab, \&

Ali, 2015

(Mahdavi,

Mahmoudi,

Anaran, \& Simchi,

2016)

de componentes: HA +
cálcicos + Fibroina+ QS

$(\mathrm{PM}=600 \mathrm{KDa}, \mathrm{DD}=85 \%)+$

nanoplata.

Nanofibras de 80-170 nm obtenidas mediante electrospinning: $\mathrm{QS}+\mathrm{CEL}$

con nanopartículas de diamante

Usos del soporte

Ensayos realizados (MND).

Crecimiento de neuritas.

Ingeniería de alimentos, campos de

bioingeniería y materiales médicos.

In vitro: Propiedades antibacterianas y

mecánicas.

In vitro: Ensayo de citotoxicidad (MTT), liberación de albúmina sérica bovina y morfología celular. Fibroblastos murinos

In vitro: Cultivo de ganglios de la raíz dorsal y tinción inmunofluorescente, viabilidad celular en andamios nanofibrosos, evaluación de morfología celular.

In vitro: Viabilidad celular. Línea de osteoblastos murinos.

In vitro: Pruebas de inmersión y concentraciones iónicas inorgánicas

Aplicaciones biomédicas.

Curación de heridas.

In vitro: Viabilidad celular (MTT) en fibroblastos murinos.
Los MWCNT incorporados mejoraron la aspereza de las membranas nanohíbridas. Los

PFNM tenían una excelente propiedad antibacteriana y superhidrofobicidad.

Las nanofibras de hidrogel (QS-g-PNIPAAm / PEO permitieron el crecimiento celular, indicativo de una citocompatibilidad apropiada.

El depósito de laminina sobre las fibras, permitió el crecimiento de las neuronas y el crecimiento de neuritas. Además, cantidades más altas de $\mathrm{LN}$ adsorbidas ayudan a promover la proliferación celular que las nanofibras de PLLA.

Los CNT / nanocompuestos de nHA con QS exhiben excelentes propiedades mecánicas, buena bioactividad, biocompatibilidad y han reunido algunas propiedades requeridas de la matriz extracelular del hueso.

Los compositos fabricados combinan los beneficios de las fases basadas en fosfatos, fibroina de seda, nanopartículas de plata, y QS Son de amplio uso en biomedicina.

Nanomateriales buenos para ingeniería de tejidos, sin embargo, se requieren estudios in vivo e investigaciones preclínicas para explorar la idoneidad de las esteras

desarrolladas para aplicaciones biomédicas.

QS: Quitosano. PM: peso molecular. PGS: Poli (glicerol sebacato). PVA: polivinil alcohol. V: viscosidad. mPa*s: milipascal-segundo. kDa: kilodaltons. MTT: Bromuro de 3-(4,5- dimetiltiazol-2-ilo)-2,5difeniltetrazol. LDL: capa por capa. HA: hidroxiapatita. PLA: ácido poliláctico. PLLA: ácido poli-L-láctico. DD: grado de deacetilación. ALG: alginato. PEO: óxido de polietileno. LDH: Lactato deshidrogenasa. PCL: policaprolactona. ALP: fosfatasa alcalina. GEL:gelatina . CNT: nanotubos de carbono. nHA: nanohidroxiapatita. CD: puntos de carbono. TPU: poliuretano termoplástico. PLGA: ácido (poliláctico-co-

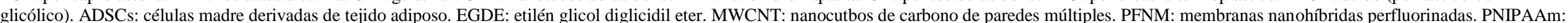
Poli(N-isopropilacrilamida). CEL: celulosa bactariana. MND: nanopartículas de diamante. 
Yeidy Viviana Arias-Andrade. Recibió su título en Quimica Industrial por la Universidad Tecnológica de Pereira en el año 2016, Durante el período 2017-2018 se vinculó como Joven Investigadora de Minciencias a los Grupos Polifenoles e Infección e Inmunidad. Su área de interés incluye la fabricación de biopolímeros basados en quitosano. Actualmente cursa estudios de Maestría en Ciencias Química en la Universidad Tecnológica de Pereira.

ORCID: https://orcid.org/0000-0001-8303-5994

Luz Angela Veloza. Recibió su título de Licenciada en Biología y Química por la Universidad de Caldas y posteriormente recibió sus títulos de Maestría y Doctorado en Ciencias- Química por parte de la Universidad del Valle, Cali, Colombia. Realizó estancia doctoral en el Instituto de Química Orgánica de la Universidad de Würburg en Alemania. Su área de interés incluye la química orgánica de productos naturales, transformación de productos naturales y su actividad biológica, así como los procesos de aprovechamiento de la biomasa. Actualmente es Directora del Grupo Polifenoles y Docente adscrita a la Facultad de Tecnologías de la Universidad Tecnológica de Pereira.

ORCID: https://orcid.org/ 0000-0001-6571-8759

Juan Carlos Sepúlveda Arias. Médico y Cirujano egresado de la Universidad de Calda y Doctor en Ciencias Biomédicas de la Universidad del Valle. Formado en Inmunología en el Centro de entrenamientos de la Organización Mundial de la Salud en Laussane, Suiza. Estuvo vinculado al Instituto de Virología e Inmunobiología de la Universidad de Würburg en Alemania durante varios años. Sus áreas de interés incluyen la Inmunología de enfermedades infecciosas, la Biología Molecular y la Biotecnología, así como la Medicina Regenerativa e Ingeniería de Tejidos. Actualmente es Director del Grupo Infección e Inmunidad y Docente adscrito al Departamento de Ciencias Básicas de la Facultad de Ciencias de la Salud de la Universidad Tecnológica de Pereira.

ORCID: https://orcid.org/0000-0002-5410-5997. 